\title{
Performance Improvement of Pumps Fed by the Variable Speed Drives
}

\author{
Valery Vodovozov (Professor, Tallinn University of Technology), \\ Ilja Bakman (Doctoral Student, Tallinn University of Technology)
}

\begin{abstract}
Speed inaccuracy decreases the pump efficiency, reliability, and energy saving. This research is devoted to the determination of the ways of accurate speed control of the pump drives operated under changeable loads. The impact of speed inaccuracy on the pump performance is studied. Based on the analysis of methods for the static accuracy improvement, the drawbacks of the traditional approaches have been shown with reference to the pumping applications. A new methodology of the slip compensation has been proposed for implementation to improve the scalar drive performance. It notably decreases the speed inaccuracy of the open-ended pumping applications. The enhanced quality of the drive control at different loading conditions has been shown on a laboratory test bench. Also, for the multi-pump systems this approach results in an additional benefit from the viewpoint of the operation around the best operation point providing a safe pump control both to exclude the pump damage and to improve the process quality.
\end{abstract}

Keywords - Pumps, motor drives, variable speed drives, sensorless control.

\section{INTRODUCTION}

The pumps belong to the class of the variable load machines the torque of which changes as a function of speed. They develop a reduced torque when driven at speeds well below the rated level and an increased torque as the speed grows. Besides the pumps, the loading systems with viscous friction, calendaring equipment, Eddy current brakes, and separately excited direct current generators feeding fixed resistance loads also fall into this group of machines. Since fans and centrifugal blowers are similar in operation to some sorts of pumps, much of them are also applicable here.

The properties of the pump as a motor load are often discussed from the viewpoint of the drive design as well as the pump start-up behavior, response to pump overload, and losses of priming fluid as well as their characteristics as the control system elements [1], [2]. Nevertheless, in multiple investigations of pumping processes, the pumping drive controllability under the changing loads has not been properly analyzed.

This paper focuses on the area of the centrifugal pumps fed by induction variable speed electric drives.

In the drives of pumps the load torque alternation results in the speed change. In this connection, traditional methods of the speed inaccuracy compensation are reputed as ineffective for pumping applications.
The purpose of this study is to determine new ways to regulate the speed of the pump motors in the conditions of changeable speeds and loads.

The paper includes the following sections. Firstly, different approaches to the variable load motor drive control are reviewed. Further, the impact of speed inaccuracy on pump performance is analyzed. Next, different methods of improving the static accuracy in the open-ended drives are compared. Then, a new methodology to decrease the speed errors is proposed and verified. The quality of the drive control at various loading conditions is explored on the laboratory test bench. Finally, conclusions are drawn.

\section{REVIEW OF CONTROL STRATEGIES FOR VARIABLE LOAD DRIVES IN RESPECT TO PUMPING APPLICATIONS}

In many variable load machines the torque is proportional to some power of the speed [3]. In the pumps, fans, and blowers the torque changes with the square of the speed and the power varies with the speed cubed throughout the range of usable speeds. Propellers in ships or airplanes also have the same type of the speed-torque characteristic. With certain type of lathes, boring machines, milling machines, steel mill coilers, etc., the hyperbolic speed-torque characteristics are associated in which the torque is inversely proportional to the speed. Additionally, in all the machines with crankshafts, particularly in reciprocating pumps and compressors, frame saws, weaving looms, rocking pumps used in petroleum industry, etc. the torque is described as a function of the crank position, i.e. the angular displacement of the motor shaft or rotor. The load of the drives used for steering ships also belongs to this category.

At closer examination, the load of the significant group of centrifugal pumps represents an enough complex composition of the above mentioned loads in varying proportions [4]. This is the reason, why the torque of these mechanisms can be resolved into two components, one of constant magnitude and the other, periodically changing depending on the speed or the angular position of the shaft. In this way, neglecting the small deviations in angle from the equilibrium position, a load can be transformed to one which varies in respect to time. This alternation may be periodic and repetitive. 
Many pumps operate under cyclically varying loads [5], [6]. Unlike the machines with continuous constant loads, such as pumps or fans operating for a long time at the same conditions, it is convenient to classify the cyclically operated pumping equipment under the following groups:

- pulsating loads, such as reciprocating pumps and compressors [7]

- impact loads, such as apparent, regular and repetitive load peaks or pulses which occur in pumps of waste applications [8]

- short-time intermittent loads, such as those occurring at almost all forms of abnormal operations [9]

Certain pumps do not strictly fall into any of the above groups. In a great deal it concerns the centrifugal pumps capable of transporting high-viscosity fluids and solid-liquid mixtures used in various industrial settings, including cement plants, sewage treatment plants, food plants, and multiple medical fields [7]. If these loads are characterized by frequent impacts of comparatively small peaks, it would be more appropriate to classify them under continuous variable loads rather than under impact loads. Sometimes, it is difficult to distinguish pulsating loads from impact loads since both of them are periodic in nature and, hence, may be expressed as a sum of sinusoidal waves of different amplitude, frequency, and phase. One and the same machine can be represented by a load torque which varies either with speed or with time. For example, a pump whose load torque is proportional to the square of the speed is also a continuous constant loading machine. Rocking pumps for petroleum have a load varying with angular position of the shaft, but can also be classified as pulsating mechanisms.

Accuracy of the pump speed adjustment at the load variations is greatly dependent on the control strategy of the variable speed electric drive. As the fast torque response is not a crucial requirement in the pumping applications, the motor speed control in such plants is usually based on the scalar voltage-frequency method [10], [11]. Because of the openended topology, the speed adjustment possibilities of the pump drive remain low enough. To improve situation, online measuring equipment is sometimes applied purposing the load assessing to estimate the temporary fluid rate of the pipeline and to make decision of its use [12]. Nevertheless, they weakly affect the pumping management [13].

Resulting from the system analysis of working conditions in pumping systems, many problems are encountered due to the speed inaccuracy at the speed-load alternation, such as hydraulic hammers, dynamic stresses in the mechanical parts, overheating in the driving motors, and other issues [1].

From this viewpoint the energy saving is a very sensitive problem [14]. Besides, the reliability of the pump also depends on the speed-load variations since the risk of cavitation and the magnitudes of hydraulic excitation forces on the impeller are minimized. If the pump is working outside the preferred operating region, the rate of mechanical wear grows. As shown in [15], operation at 70 or $115 \%$ of the best efficiency point tenfold decreases the characteristic life of the pump. In these conditions, the safe pump control should be considered as an effective instrument both to exclude the pump damage and to improve the pumping quality [16].

Additionally, as shown in [17], [18], the speed errors decrease the accuracy of the pressure calculation in the sensorless control systems.

\section{ANALYSIS OF SPEED INACCURACY IMPACT ON PUMP PERFORMANCE}

To optimize most of pumping features, it is reasonable that the major pump operation occurs at its best efficiency point (BEP). Resulting from the system analysis of working conditions in pumping systems, many problems are encountered as a result of the speed inaccuracy at the load alternation around the BEP, such as hydraulic hammers, dynamic stresses in the mechanical parts, overheating in the driving motors, and other issues [1]. The reliability of the pump also depends on the BEP since the risk of cavitation and the magnitudes of hydraulic excitation forces on the impeller are changed. If the pump is working outside the preferred operating region, the rate of mechanical wear grows. As shown in [15], operation at 70 or $115 \%$ of the BEP tenfold decreases the characteristic life of the pump. In these conditions, the safe pump control should be considered as an effective instrument both to exclude the pump damage and to improve the pumping quality [16]. Besides, as shown in [17], [18], the speed errors decrease the accuracy of the pressure calculation in the sensorless control systems.

Nevertheless, the most sensitive problem from this viewpoint concerns the energy saving.

As follows from [19], the total contemporary world electricity installed capacity is about $5000 \mathrm{GW}$. The pumps, fans, and compressors consume about half of this volume, $90 \%$ of which relates to the centrifugal pumps discussed in this study. As electric drives consume $70 \%$ of all the generated energy, this means that even $1 \%$ of energy economy amounts to $23 \mathrm{GW}$. To understand the meaning of this figure, it is enough to compare it with the national electricity installed capacity of such countries as Estonia (2,7 GW), Slovenia (3,2 GW) or Germany (153 GW).

According to [14], the pump efficiency can drop significantly because of the speed inaccuracy. Typically, a pump motor is considered to be operating at the maximum efficiency when the load torque is about $75 \%$ of the rated value [4]. The efficiency of the motor drops along with its speed is decreasing due to increased losses. Also, if the motor is driven at a low speed, it has a decreasing effect on the efficiency of a frequency converter feeding the motor. Additionally, as the pump load torque curve has a squared relationship with the rotational speed, the resulting efficiency tends to be significantly decreased when a centrifugal pump is driven at a low speed. Therefore, to optimize the energy consumption based on efficiency, it is reasonable that the major way to operate the centrifugal pump is at its BEP. 


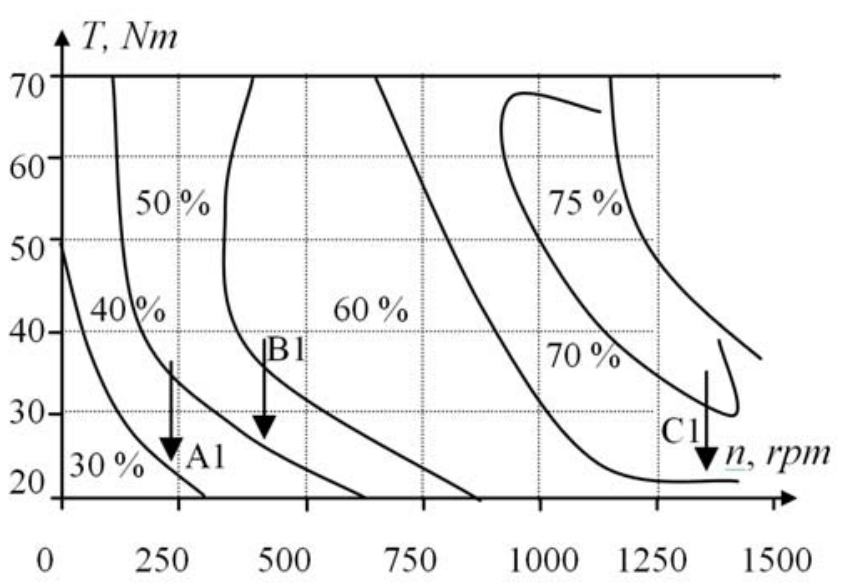

(a)

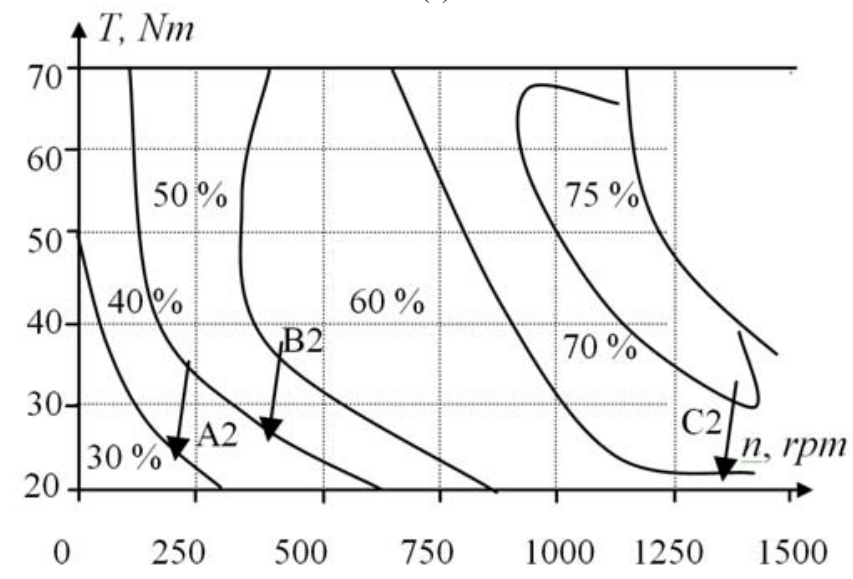

(b)

Fig. 1. Efficiency maps of induction motor in torque-speed reference frame built on the basis of [14]. The arrows show efficiency change under the torque drop at the stable speeds (a) and at the $10 \%$ speed inaccuracy (b).

To analyze the impact of the speed inaccuracy on the pump efficiency associated with the changeable speed-torque conditions, the efficiency maps were adopted instead of using particular operating points, such as the rated power at the rated torque and the rated speed. The required map shown in Fig. 1 was built by processing the efficiency data from [14] for the set of three-phase induction motors.

Using this map, two situations were explored, both for the $30 \%$ drop of torque $T$. The former study, shown in Fig. 1 a, relates to the stable (load-independent) speed levels of 250 , 400, and $1350 \mathrm{rpm}$ respectively. The latter study, shown in Fig. $1 \mathrm{~b}$, concerns the load-dependent situation where the speed drops by $10 \%$ due to the speed control inaccuracy. The arrows $\mathrm{A} 1, \mathrm{~B} 1, \mathrm{C} 1$ and $\mathrm{A} 2, \mathrm{~B} 2$, and $\mathrm{C} 2$ illustrate these situations. According to analysis, in the first case the efficiency drops by $10 \%$ (A1, B1) at low speeds, or $5 \%(\mathrm{C} 1)$ at high speeds, whereas in the second case the efficiency drops by $20 \%$ (A2, B2), or $15 \%$ (C2). From this analysis, a necessity to the speed stabilization follows with obviousness.

\section{Estimation of the ScAlar SpeEd Control Methods}

The pump is fed by a squirrel cage induction motor with the following variables and parameters:

$\mathrm{U}$ - phase supply voltage,

$\mathrm{T}-$ motor torque,

$\omega=\frac{\pi n}{30}-$ angular frequency of the motor shaft,

$\omega_{0}=\frac{2 \pi f}{p}=\frac{\pi n_{0}}{30}-$ angular frequency of the supply voltage,

$\mathrm{n} 0$ - desired rotational speed of the motor shaft,

$\mathrm{n}$ - actual motor speed,

$\mathrm{f}$ - supply frequency,

$\mathrm{p}$ - pole pair number,

$\mathrm{R} 1$ - stator winding resistance,

$\mathrm{R} 2$ - rotor winding resistance,

$\mathrm{Xk}$ - short circuit reactance.

This is a well-known fact [3], [11], [20] that some difference $s$ exists between the desired and actual speeds of an induction motor called an absolute slip,

$$
s=\omega_{0}-\omega .
$$

The slip comes from the inefficiency of the motor rotor to completely convert its magnetic field into an induced magnetic field in the rotor. In accordance with the Kloss' formula

$$
T=\frac{2 T_{b}(1+\varepsilon)}{\frac{s}{s_{b}}+\frac{s_{b}}{s}+2 \varepsilon},
$$

the slip can be described as a complex functions of the drive parameters and operational conditions:

$$
s=\frac{R_{2}}{R_{1}} \varepsilon \omega_{0}\left(\frac{T_{b}}{T}(1+\varepsilon)-\varepsilon-\sqrt{\left(\frac{T_{b}}{T}(1+\varepsilon)-\varepsilon\right)^{2}-1}\right),
$$

where

$$
\begin{gathered}
\varepsilon=\frac{R_{1}}{\sqrt{R_{1}^{2}+X_{k}^{2}}}, \\
\text { but } T_{b}=\frac{3 U^{2} \varepsilon}{2 \omega_{0} R_{1}(1+\varepsilon)}
\end{gathered}
$$

breakdown torque of the motor.

In [21] a more accurate analytical method was proposed to determine the mechanical characteristics of the asynchronous motors upon the changing supply frequency. Based on (2), the method enhances calculation of the relative magnitudes of the torques under respective slips in the starting and operational parts of the mechanical characteristics as well as under the rated motor performance.

In any case, the absolute slip depends primarily on the speed and torque values, which are closely connected and heavily alternated in the pumps fed by the variable speed drives. As the speed of the pump changes in proportion to the load torque, this means that the rotor slip alternates in the 
same proportion. The full-load slip may vary from less than 1 percent in high-power motors to more than 5 percent in fractional-power machines. With small drive systems the rated slip is about $7 \ldots 15 \%$ of $\mathrm{n}_{0}$ while the larger drives have the slip of $2 \ldots 5 \%$. These variations may cause load-sharing problems when motors of different sizes are connected mechanically. At low loads the sharing is approximately correct, but at full loads the motor with lower slip takes a higher share of the load than the motor with higher slip.

Since the slip is a common problem with standard motors, several options exist to reduce its effects

Choosing of an oversized induction motor is the simplest way to reduce the slip. Larger motors typically have a lower slip value to begin with, and slip gets smaller with a partial, rather than full, motor load. The disadvantage of the motor overrating is that larger motor has higher energy consumption, which increases investment and operation costs.

In [22] a slip compensator, based on fuzzy logic incremental controller has been developed to improve the steady state performance of efficiency-optimized three-phase induction motor drive system. Efficiency optimization here was accomplished by using a search controller manipulating the value of stator voltage, at which the efficiency is at its maximum for any operating point. Unfortunately, the proposed controller reduces the slip occurring at low frequencies and light loads, which are not typical for pumping applications. Another drawback of the described solution is that slip compensation reduces the maximum efficiency of the system.

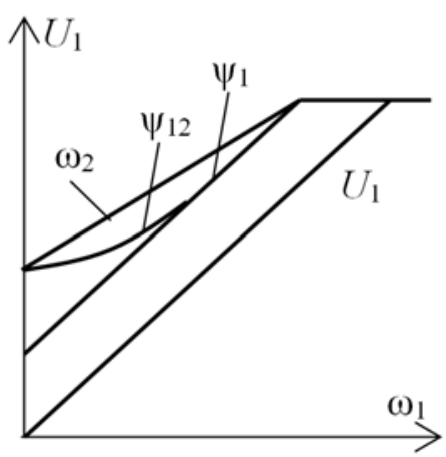

(a)

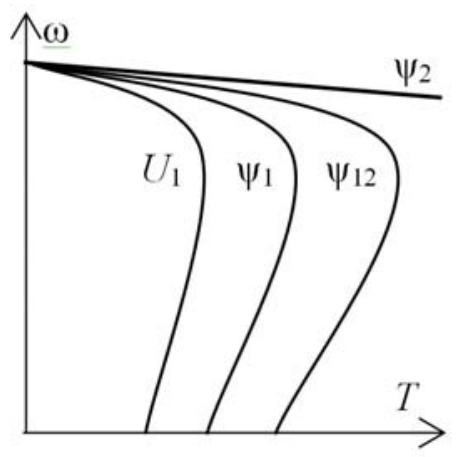

(b)

Fig. 2. Control (a) and mechanical (b) curves of the flux-frequency control.
Traditionally, to obtain the load-independent speed-torque characteristics in the open-ended induction motor drive, an adjustment of the stator electromotive force (EMF) $E_{1}$ along with the stator angular frequency $\omega_{1}$ is recommended [3], [10], [11]. In this way the motor stator flux $\psi_{1}$ can be fixed as:

$$
\psi_{1}=\frac{E_{1}}{\omega_{1}}=\text { const } .
$$

This mode of the variable speed control is known as a scalar stator flux-frequency control (lines $\psi_{1}$ in (Fig. 2)). To obtain the accurate EMF adjustment, the stator EMF $E_{1}=U_{1}-I_{1} R_{1}$ is to be calculated by sensing the stator current $I_{1}$ and subtracting of the current-resistance product $I_{1} R_{1}$ from the voltage reference $U_{1}^{*}$.

Without EMF control (lines $U_{1}$ in Fig. 1), at low speeds as well as in the small motors and at large loads, the ohmic voltage drop $I_{1} R_{1}$ notably affects the control quality by lowering the motor magnetic flux and overload capacity as the frequency decreases. It must be mentioned that the EMF calculation is rather complicated due to the $R_{1}$ dependence on the temperature instability.

An actual torque produced, however, is non-linearly connected with the speed and load, therefore the simple EMF control is ineffective at low speed levels. To further increase the speed control accuracy at changeable loads, both the stator voltage drop and the magnetization reluctance are to be stabilized, i.e. the constancy of the effective flux $\psi_{12}$ is likely to be employed. This approach is known as an effective fluxfrequency control or boosting. The lines $\psi_{12}$ in Fig. 2 illustrate the boosting mode.

According to (3), as soon, as the effective flux is stabilized, the slip now depends only on the torque-frequency ratio because the supply voltage follows the frequency and the impedance is compensated. Additional control improvement could be achieved using direct slip compensation. Better adjustment can be obtained here by summing the desired speed signal with an error equal to the actual slip value. Commonly, this method has some restrictions.

Usually, to measure the slip, a speed sensor has to be fitted to the motor shaft, which is not suitable for the scalar openended control, especially in the multi-motor pumping applications.

Instead of the speed, the torque could be sensed. The slip control loop in this case is actually the torque control loop while considering the torque practically proportional to the slip in the control range involved as follows from the curves $\psi_{2}$ on the motor control and speed-torque characteristics in Fig. 2. Unfortunately, as it was shown above, the torque of pumps changes with the square of the speed, therefore this method is also unsuitable for the scalar control. For this reason, the traditional slip compensation methods accepted in the variable speed drives of major companies are not effective for pumps. They assume the compensation as a constant parameter, which a customer must predefine before operation whereas in pumping processes it is a function of speeds and torques. 


\section{V.Proposed METHOdOLOGY OF THE SLIP COMPENSATION}

To provide the slip compensation in the load-speed changeable sensorless pumping system, the following methodology has been developed.

First, in the system tuning stage, the lookup speed-slip table is generated for the pumping application. As the speed-torque characteristics of pumps depend on the quality of the pumping matter (temperature, viscosity etc...), all the particular pumping equipment requires its own lookup table prepared before the process startup. At this step, the pumps are temporary equipped with the speed sensors and an experiment is executed, in which the pumps accelerate smoothly from zero to the maximal speed. One example of the acceleration diagram obtained from experimentation is shown in Fig. 3.

From this diagram, using (1) the speed-slip relation is estimated and registered in the lookup table. To this aim, the full speed range is divided by the sectors in which the average slip values are calculated. Also, the speed-torque and torqueslip dependences can be analyzed here. In Fig. 4, compensation signals are shown for the lookup table consisting of ten cells. Each compensation level represents an average slip of the particular sector.

Such compensation is effective only in the traditional steady-state pumping conditions. Any time, when the reference changes quickly (at startup and breaking, for example), compensation is disconnected.

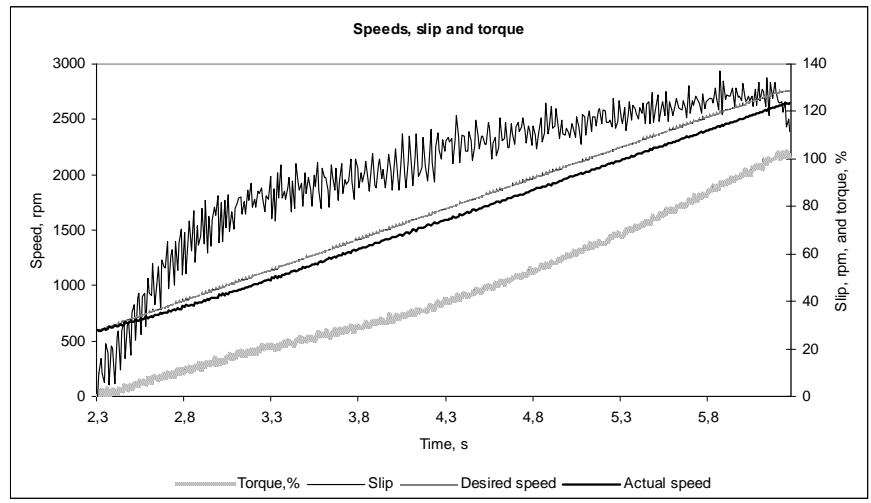

Fig. 3. Example of the drive acceleration diagram

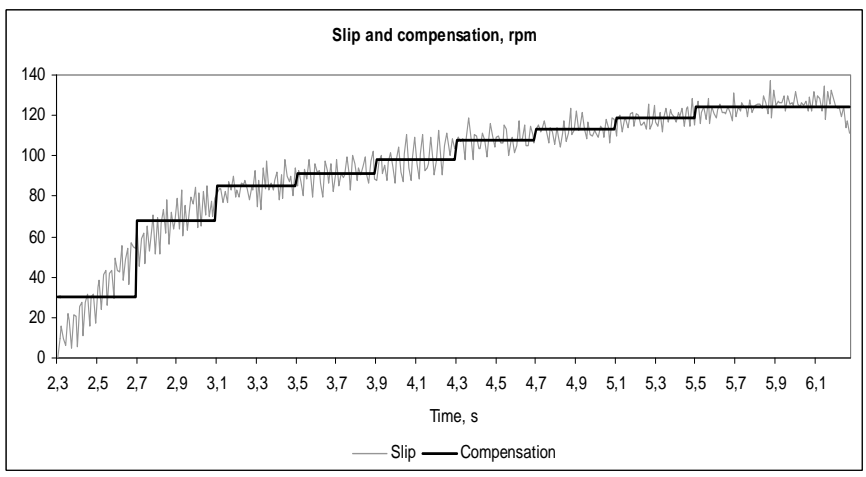

Fig. 4. Replacement of slip with compensation levels from the lookup table.

\section{IMPLEMENTATION OF SLIP COMPENSATION}

As soon as the lookup table is prepared, the slip compensation can be implemented. For an experimental study of the pump drive, the test bench was used equipped with five centrifugal pumps EBARA CDX 120 fed by the frequency converters ABB ACQ 810. Every motor is of the rated power $0,9 \mathrm{~kW}, 400 \mathrm{~V}, 3 \mathrm{~A}, 2760 \mathrm{rpm}$. The actual speeds, torques, and powers of the driving motors were estimated with the help of the ABB DriveStudio toolkit. The description of the basic topology and composition of the experimental setup can be found in [8].

During the system operation in the sensorless mode, accordingly the desired speed of the motor and the averaged slip is acquired from the lookup table and used as the slip compensation signal by adding it to the desired speed (Fig. 5).

The following equation is used to specify the rule for the proposed control:

$$
\omega_{1}^{*}=\frac{\pi\left(n_{0}+s\right)}{30} .
$$

In Fig. 6 the speed and slip after compensation are shown.

Thanks to the compensation, the average slip has 6,5 times decreased, i.e. from $96 \mathrm{rpm}(6 \%)$ in Fig. 3 to $15 \mathrm{rpm}(0,9 \%)$ in Fig. 6.

In the multi-pump applications this approach results in an additional benefit in operation around the BEP because it can provide the safe pump control both to exclude the pump damage and to improve the process quality.

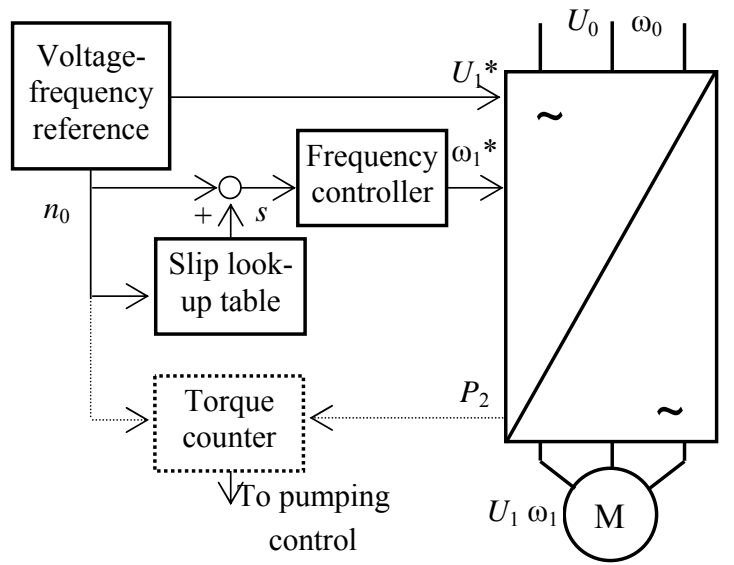

Fig. 5. Circuit diagram of the sensorless slip compensation. Dotted components relate to the system with torque estimation.

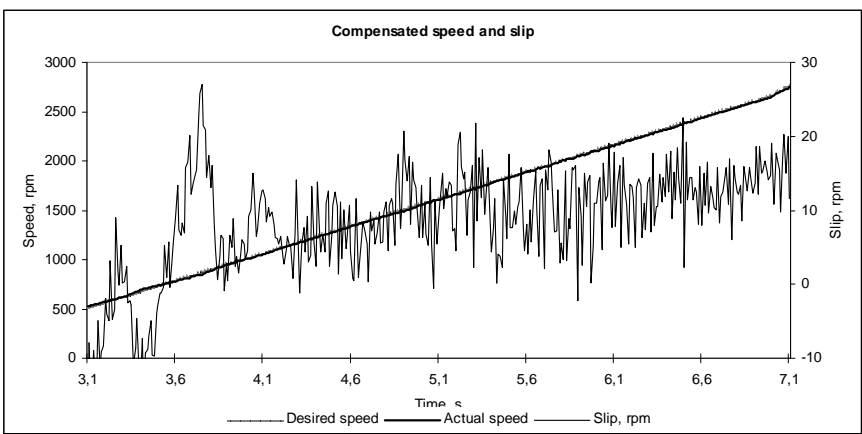

Fig. 6. Motor speeds and slip after compensation. 


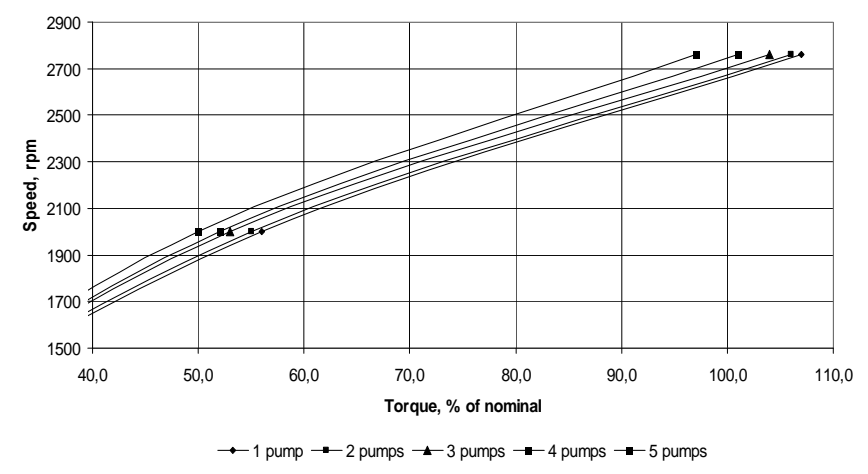

Fig. 7. Speed-torque characteristics of 5 pumps Ebara CDX 120.

To obtain this, take into account that in the variable speed drives of major companies, like ABB, Siemens, Sew EuroDrive, etc. the load torque $T$ can be estimated in every speed as a parametric function of electromagnetic torque $T_{2}$, idle torque $\Delta T$, and electric power $P_{2}$, for instance as follows:

$$
T=T_{2}-\Delta T ; \quad T_{2}=\frac{P_{2}}{n_{0}} .
$$

Now, the system design stage foresees the generation of an additional lookup torque-speed table. To prepare it, two, three, four, and five pumps were running step by step. From each running, the desired speed $n_{0}$ and the developed torque $T$ were acquired, and a new lookup table was prepared. An example of the experimental speed-torque diagrams obtained from the ABB multi-pump setup is shown in Fig. 7.

Since the new lookup table is designed on the basis of Fig. 7, the actual torque of the pump motor can be estimated along with the desired speed as shown in Fig. 5 by dots.

Using this approach, the number of pumps which provides the highest efficiency can be found. For example, as follows from Fig. 6, to develop the torque of $50 \%$ both single pump operating at $1850 \mathrm{rpm}$ and five pumps at $2000 \mathrm{rpm}$ can be used. Conversely, to operate at $2700 \mathrm{rpm}$, the customer can choose between a single pump used at $104 \%$ load and five pumps with $94 \%$ load.

\section{CONCLUSIONS}

Speed inaccuracy decreases pump efficiency, reliability, and energy saving. Based on the analysis of methods for the static accuracy improving, the drawbacks of the traditional approaches have been shown with reference to the pumping applications. A new methodology of the slip compensation has been proposed for implementation in the scalar drive to improve performance. It notably decreases the speed inaccuracy of the open-ended pumping applications. The enhanced quality of the drive control at different loading conditions has been shown on the laboratory test bench. Also, for the multi-pump systems this approach results in an additional benefit from the viewpoint of the operation around the best operation point providing the safe pump control both to exclude the pump damage and to improve the process quality.

\section{ACKNOWLEDGMENTS}

This research work has been supported by Estonian Ministry of Education and Research (Project SF0140016s11) and European Social Fund (project "Doctoral School of Energy and Geotechnology II").

\section{REFERENCES}

[1] H. L. Stewart, Pumps, Indianapolis: Sams, 1977, 465 p.

[2] A. A. Divona and A. J. Dolan, "Electric motors and motor controls," In: Karassik, I. J., Krutzsch, W. C., Fraser, W. H. and Messina, J. P. (Eds.), Pump Handbook, pp. 6.3-6.25, NY: McGraw Book Company, 1985.

[3] B. K. Bose, Modern Power Electronics and AC Drives, Englewood Cliffs, NJ: Prentice-Hall PTR, 2001.

[4] I. I. Ionel, Pumps and Pumping with Particular Reference to VariableDuty Pumps, Amsterdam: Elsevier, 1986, 715 p.

[5] Y. V. Smirnov, "Improving asynchronous electric drives of machines with a periodically varying load," Russian Electrical Engineering, 2007, vol. 78 , no. 6, pp. $287-290$.

[6] L. Peretti and M. Zigliotto, "Identification of mechanical load for electrical drives commissioning - Labelling machine case study," International IEEE Conference EUROCON 2009, St.-Petersburg, Russia, 2009, pp. 797-803.

[7] K. Saito, Y. Hirayama, Y. Kimura and T. Nakamura, "Development of a peristaltic pump based on bowel peristalsis improvement of closing area rates and suction pressure measurement," The 4th IEEE RAS/EMBS International Conference on Biomedical Robotics and Biomechatronics, Roma, Italy, 2012, pp. 949-954.

[8] I. Bakman and V. Vodovozov, "Multi-Pump Control Applications for ABB ACQ 810 Industrial Drive," 12th International Symposium "Topical Problems in the Field of Electrical and Power Engineering." Doctoral School of Energy and Geotechnology II, Kuressaare, Estonia, 2012, pp. 37-44.

[9] H. H. Benjes and W. E. Foster, "Sewage treatment," In: Karassik, I. J., Krutzsch, W. C., Fraser, W. H. and Messina, J. P. (Eds.), Pump Handbook, pp. 9.23-9.39, NY: McGraw Book Company, 1985.

[10] N. Mohan, First Course on Power Electronics and Drives, Minneapolis, MN: MNPERE, 2003, $248 \mathrm{p}$.

[11] V. Vodovozov, Electric Drive Systems and Operation, London, UK: Bookboon, 2012, $102 \mathrm{p}$.

[12] É. Hajnal, G. Lakner, P. Ivanics, Z. Molnár and J. Lakner, "Real time control system for industrial waste water management," 16th International Conference on Intelligent Engineering Systems, Lisbon, Portugal, 2012, pp. 429-433.

[13] O. S. Ebrahim, M. A. Badr, A. S. Elgendy, and P. K. Jain, "ANN-based optimal energy control of induction motor drive in pumping applications," IEEE Transactions on Energy Conversion, v. 25, no. 3, 2010, pp. 652-660.

[14] P. Angers, "Variable frequency drive testing," Motor Efficiency Performance Standards, Sydney, Australia, 2009.

[15] T. Ahonen, Monitoring of Centrifugal Pump Operation by a Frequency Converter, Doctoral Thesis, Lappeenranta: LUT, 2011, 134 p.

[16] Z. Chenghu, W. Haiyan and S. Dexing, "Flow resistance and energy analysis of urban sewage delivery heat transfer system," $3^{\text {rd }}$ International Conference on Measuring Technology and Mechatronics Automation ICMTMA 2011, Shanghai, China, 2011, pp. 234-237.

[17] V. Vodovozov and I. Bakman, "Control of liquid density to prevent abnormal pumping performance," 7th WSEAS International Conference on Waste Management, Water Pollution, Air Pollution, Indoor Climate WWAI 2013, Lemesos, Cyprus, 2013, pp. 217-222.

[18] I. Bakman, "Implementation and testing the sensorless pressure measurement of centrifugal pumps," 13th International Symposium "Topical Problems in the Field of Electrical and Power Engineering." Doctoral School of Energy and Geotechnology II, Pärnu, Estonia, 2013, pp. $132-138$.

[19] International Energy Statistics, [Online]. Available: http://www.eia.gov/cfapps/ipdbproject/IEDIndex3.cfm?tid=2\&pid=2\&ai $\mathrm{d}=7$.

[20] J. Böcker and S. Mathapati, "State of the art of induction motor control," IEEE International Electric Machines \& Drives Conference IEMDC 2007, Antalia, Turkey, 2007, pp. 1459-1464. 
[21] J. Buksnaitis, "Analytical determination of mechanical characteristics of asynchronous motors by varying the electric current frequency," Electronics and Electrical Engineering, Kaunas: Technologija, 2011, $6(112)$, p. 3-6.

[22] H. Sarhan, R. Issa, M. Alia and J. M. Assbeihat, "Slip compensation in efficiency-optimized three-phase induction motor drive systems," Intelligent Control and Automation, 2011, 2, pp. 95-99.

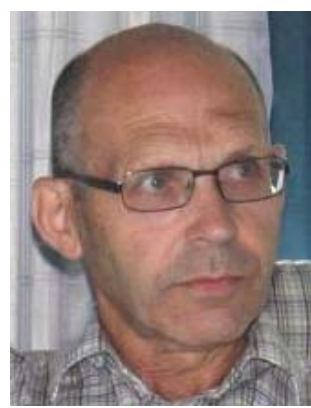

Valery Vodovozov received his Candidate of Science degree and Associate Professor and Senior Researcher academic titles in Electrical Engineering from St. Petersburg Electrotechnical University, Russia. His areas of expertise cover electric drives and power electronics, application of object-oriented technologies in industry, and Web-based education.

$\mathrm{He}$ is a professor of Tallinn University of Technology. Earlier he served as an Associate Professor and Senior Researcher in St. Petersburg Electrotechnical University, Russia visiting researcher in Scientific Research Laboratory of Ford Motor Company, USA; lectured at St. Petersburg Institute for Extra Professional Education, St. Petersburg University of Economics and Finance, St. Petersburg Customs
Academy, and taught regular academic courses in institutional, industrial and customs training centers of Russia, USA, and Estonia.

Valery Vodovozov is a member of IEEE, International Institute of Informatics and Systemics (IIIS), Global Research Alliance of Texas Institute of Science (TxIS GRA), and Estonian Society of M. H. Jacobi.

Postal address: Ehitajate tee 5, Tallinn, 19086, Estonia.

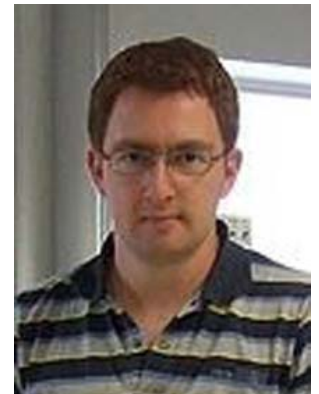

Ilja Bakman received his Bachelor and Master degrees from Tallinn University of Technology. His field of interests includes electric drives of pumping systems, software development for automation systems, DSP, developing the automated tests for software products, integration of automation system elements, creation and optimization of algorithms for pumping applications in the field of water and waste water processing, support, consulting, and trainings for service engineers.

Currently he is ABB AS engineer of automation and a $\mathrm{PhD}$ student of Tallinn University of Technology. Before, he was a process engineer at AMS Electronics.

Postal address: Ehitajate tee 5, Tallinn, 19086, Estonia. 